\title{
Acquisition of Kowledge Data by Analyzing Natural Language
}

\author{
Yasuhito Tanaka \\ Himeji College \\ 1-1-12 Shinzaike Honmachi \\ Himeji City Hyogoken \\ 670 JAPAN
}

\section{Introduction}

Automatic identification of homonyms in kana-tokanji conversions systems and of multivocal words in machine translation systems cannot be sufficiently implemented by the mere combination of grammer and word dictionaries. This calls for a new concept of knowledge data. What the new knowledge data is and how it can be acquired are mentioned in the paper. In natural language research, active discussion has been make within the framework of knowledge and samples of knowledge.

2. Phases of Natural Language Study and Knowledge Data

[phase 1]

In the initial phase when natural language had not been fully clarifjed, the authors made an attempt to analyze the language with the help of word dictionaries and grammar alone, and to build a new system from the results. We, however, encountered a great number of homonyms in kana-to-kanji conversion, as well as multivocal words and other problems in machine translation. This was because the grammar and dictionaries were too simple. In other words, $\omega_{i} \rightarrow f_{\mathrm{T}}\left(\omega_{i}\right)$

where $\omega_{i}$ is a word and $f_{\mathrm{T}}$ is a conversion system.

[phase 2]

There are some possible means for solving the problems encountered in Phase 1. They include the following: analyze the word frequency, limit the number of words in use, limit the contents of the words in use, limit the fields and limit sentences. This helps remove, or at least minimize, homonyms, multivocal words and ambiguities. A system with limitations, however, involves too many difficulties to be readily accepted.
Sho Yoshida

Kyushu University

6-10-1 Hakozaki Higashiku

Fukuoka City Fukuokaken

812 JAPAN

[phase 3]

In vocabulary, no words are used independent1y. One should define and use words by clarifying the characteristics, coverage and conditions of words. This may be expressed as follows:

$$
\begin{aligned}
& \omega_{i} \mid P_{i 1}, P_{i_{2}}, P_{i 3}, \cdots, P_{i n} \\
& \rightarrow f_{T}\left(\omega_{i} \mid P_{i_{1}}, P_{i_{2}}, \ldots, P_{i n}\right)=y j
\end{aligned}
$$

where $\omega_{i}$ is a word; and $P_{i 1}, P_{i 2}, \cdots, P_{i n}$ are the limitations of $\omega_{i}$. The set of $P_{i 1}, P_{i 2}, \cdots, P_{i n}$ should be low in number and simple.

Different approaches have already been proposed as to what is necessary for clarifying the conditions for the coverage of words in Phase 3. Some examples are semantic markers, semantic categories and thesaurus. Nowever, the authors put emphasis on the relationship of words, though recognizing such other forms of approach.

[Knowledge Obtained from Words]

Words and sentences provide knowledge shown in Table 1, from simple to complicated in order. How much of them are usable as dictionaries (books)? How much are usable as machine-readable dictionaries?

In Table 1, machine-readable dictionaries have already been established for 1. "Word-related Attributes". Classified vocabulary lists have been prepared for Thesaurus structure in 2, but there is almost nothing for the other attributes.

Therefore, an attempt to build a sophisticated system would be unsuccessful if no basic knowledge data is available. 
Table 1. Knowledge obtained from Words

1 Word-Related Attributes

1.1 Word Attributes

Words, parts of speech, pronunciation, accent and kanla representation

1.2 Long Unit Words and Technical Terms

2 Word-to-Word Attributes (1) (Preconditions)

2.1 Broader:/Narrower Rank Relationship (Thesaurus Structure)

2.2 Antonyms and Negatives

2.3 Partial/Whole Relationship

2.4 Sequential Relationship

2.5 Comparative Relationship (size, height)

3 Word-to-Word Attributes (2) (Preconditions)

3.1 Case Relationship

3.2 Relationship Based on the Synchronism of Sentence Components

3.3 Idiomatic expressions

4 Word-to-Word Attributes (3) (Preconditions)

4.1 Association-Based Relationship

5 Sentence Re1ationship

5.1 Sentence-to-Word Re1ationship

5.2. Sentence-to-Sentence Linkage

3. Is it Possible to Define Word Coverage and Conditions?

Since every word may be linked with an infinite number of words, one may wonder if it is impossible to define word coverage and conditions. It would also take a great deal of time and trouble to examine a11 the words that are almost infinite in number. If, however, one actually examines some of the words, we will fine that every word is linked with a limited number of words.

Table 2. Each Word is Linked with

$$
\text { a Limited Number of Words }
$$

$\begin{array}{llll}001 & \text { Denwa o kakeru } & 018 & \text { Denwa o migaku } \\ 002 & \text { Denwa o kiru } & 019 & \text { Denwa o ukeru } \\ 003 & \text { Denwa o mochiageru } & 020 & \text { Denwa o tochosuru } \\ 004 & \text { Denwa o kowasu } & 021 & \text { Denwa o kakenaosu } \\ 005 & \text { Denwa o nigiru } & 022 & \text { Denwa o motsu } \\ 006 & \text { Denwa o motsu } & 023 & \text { Denwa o motaseru } \\ 007 & \text { Denwa o kairyosuru } & 024 & \text { Denwa o kiku } \\ 008 & \text { Denwa o tsukuru } & 025 & \text { Denwa ga naru } \\ 009 & \text { Denwa o seisakusuru } & 026 & \text { Denwa o tsutaeru } \\ 010 & \text { Denwa o kumitateru } & 027 & \text { Denwa de hanasu } \\ 011 & \text { Denwa o kaisetsusuru } & 028 & \text { Denwa de renrakusuru } \\ 012 & \text { Denwa o hiku } & 029 & \text { Denwa ni deru } \\ 013 & \text { Denwa o tekkyosuru } & 030 & \text { Denwa no koe } \\ 014 & \text { Denwa o uru } & 031 & \text { Denwa no buhin } \\ 015 & \text { Denwa o hanbaisuru } & 032 & \text { Denwa no kane } \\ 016 & \text { Denwa o kau } & 033 & \text { Denwa no ryokin } \\ 017 & \text { Denwa o konyusuru } & 034 & \text { Denwa no beru } \\ & & \ldots . . & \text {............ }\end{array}$

Take the word, "denwa" (meaning telephone in English) as example. It has a 1 imited number of characteristics such as a means of communication, a substance, a place, and so on. The function as a means of communication and the characteristics of the telephone are unique to the telephone.

In this particular meaning, it is a simple and finite task to count the different relationships between words. It is, however, extremely difficult to count the word-to-woyd relationships for the general meanings such as a substance and a place. However, it is possible to organize the major relationships without much effort.

The only approach would be to tabulate the words having peculiar relationship to specific words and the relationship between specific words and words of high usage frequency, and thus use system-preset defaults for other words.

Such words as "takai" (meaning high) and "utsukushii" (meaning beautiful) are used frequently. In some cases, therefore, it is difficult to determine the coverage and conditions of individual words. Such cases must inclucle general grammar and the meaning of words most frequent $1 y$ used in "takai" and "utsukushil" must be defined in the word-to-word relationships together with the conditions of any special meanings of high and beautiful. Words of low frequency and high frequency are dependent on individual rules (conditions based on word-to-word relationship) and general grammar.

4. Aquisition of Knowledge data

4-1 Method by extracting and segmenting Kanji strings

Authors and S. Mizutani of Tokyo Women's Christian College mechanically extracted the four-character kanji strings from some JICS' abstract files, extracted meaningful concept combinations from them, and classified them into 45 categories. Thus, after examing 78,000 four character kanji strings, 28,000 different kinds of knowledge data was obtained from 32,000 types of kanji strings in net. This approach is promising because it allows easy expansion in quantity and consists mainly of mechanical processing. In addition, an attempt to reorganize a total of 887,000 data, 200,000 in net, is under way. An attempt to apply the same idea to the three and five character kanji strings is also being made. 
[Example]

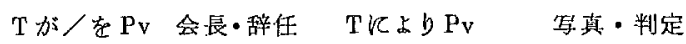

T ga/o Pv kaicho-jinin T ni yori Pv shashin-hantei

4-2 Aquisition of knowledge data based on word-toword synchronism

In this case, Japanese sentences should be analyzed to extract connections and obrain knowledge data. Analysis, however, takes a great deal of time and involves ambiguity. This suggests an approach which simply extracts independent words handles them, if present in the same sentences, as candidates for knowledge data because of mutual relation, and extracts meaningful knowledge data based on frequency and manual procedure.

[Example]

少年ががールを投げる

A boy throws a ball.
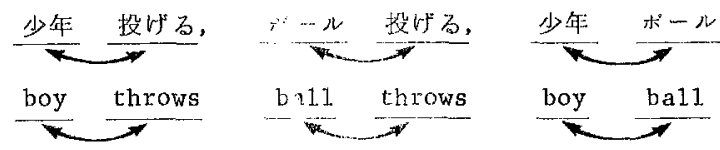

To extract such re! ' 'ships and reorganize them sequentially.

4-3 Aquisition of knowledge by syntax analysis

This is a method which analyzes the sentences that are input with word dictionaries and grammar, builds syntax elements, extracts connections from them, and thus, extracts knowledge data. This method is theoretically pertinent but not practical because it leaves the problem of a tremendous increasing number of syntax trees. It would be very effective if a great amount of knowledge data were available and some of the ambiguities from syntax analysis were reduced. Since, in Japanese sentences, long-unit terms are used as they are, it is also necessary to sub-categorize them automatically. Otherwise, a variety of knowledge data would result and it would be troublesome to systematize knowledge data.

XX daigaku (university), XY daigaku $\Longleftrightarrow$ daigaku

$$
\text { general terms basic concept term }
$$

This sort of concept was presented by some manufacturers engaged in the research and development of machine translation. It is however only in the planning stage. There has been no news reporting that knowledge data is effectively available.

Fig. 2. Method of Obtaining Knowledge

by Syntax Analysis

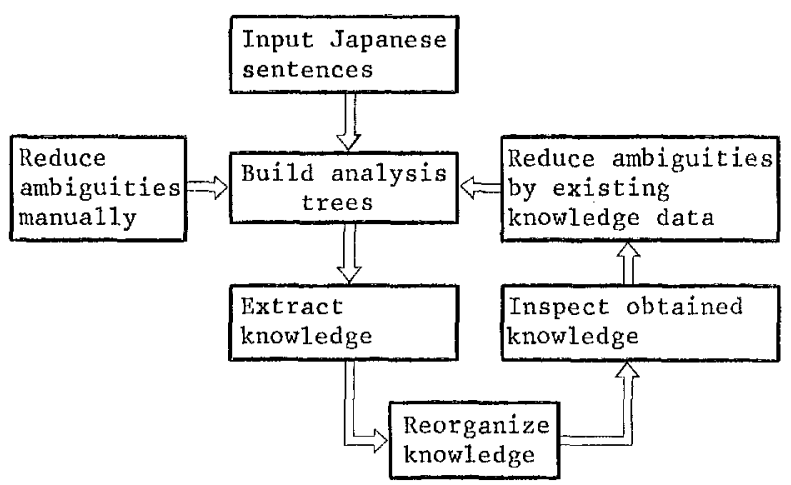

5. Applications of Knowledge Data

(1) To develop high quality Japanese word processors.

(2) To improve the quality of machine translation.

(3) To reduce the ambiguities of syntax analysis.

(4) To apply knowledge data in handwritten character and voice recognition.

6. Conclusion

The systemization, aquisition and construction of knowledge data are a step towards the next jump in Japanese processing systems. Indeed, the knowledge data still has a number of problems to be solved, but prospects for the future are rather bright.

References

(1) Inanaga \& Konishi, Terms for Computer-Based Processing of Kana Characters AL 76-39 (in Japanese). Material of Engineering Workshop of: Electronic Communications Society, 1976

(2) Shizuo Mizutani, Overview of Word Structure (in Japanese). Iwanami Japanese Language Dictionary (3rd Edition), March 1980

(3) Masaaki Yamanashi, Meaning and Knowledge Structure: Theoretical Study of Meaning Expression Mode1s from Linguistics (in Japanese). Mathematical Science No. 240, June 1983. 